\title{
Sparsening and Temporal Sharpening of Olfactory Representations in the Honeybee Mushroom Bodies
}

\author{
Paul Szyszka, ${ }^{1}$ Mathias Ditzen, ${ }^{1}$ Alexander Galkin, ${ }^{1}$ C. Giovanni Galizia, ${ }^{2}$ and Randolf Menzel ${ }^{1}$ \\ ${ }^{1}$ Institut für Biologie-Neurobiologie, Freie Universität Berlin, Berlin, Germany; and ${ }^{2}$ Department of Entomology, University of California, \\ Riverside, California
}

Submitted 18 April 2005; accepted in final form 9 July 2005

\begin{abstract}
Szyszka, Paul, Mathias Ditzen, Alexander Galkin, C. Giovanni Galizia, and Randolf Menzel. Sparsening and temporal sharpening of olfactory representations in the honeybee mushroom bodies. $J$ Neurophysiol 94: 3303-3313, 2005. First published July 13, 2005; doi:10.1152/jn.00397.2005. We explored the transformations accompanying the transmission of odor information from the first-order processing area, the antennal lobe, to the mushroom body, a higherorder integration center in the insect brain. Using $\mathrm{Ca}^{2+}$ imaging, we recorded activity in the dendrites of the projection neurons that connect the antennal lobe with the mushroom body. Next, we recorded the presynaptic terminals of these projection neurons. Finally, we characterized their postsynaptic partners, the intrinsic neurons of the mushroom body, the clawed Kenyon cells. We found fundamental differences in odor coding between the antennal lobe and the mushroom body. Odors evoked combinatorial activity patterns at all three processing stages, but the spatial patterns became progressively sparser along this path. Projection neuron dendrites and boutons showed similar response profiles, but the boutons were more narrowly tuned to odors. The transmission from projection neuron boutons to Kenyon cells was accompanied by a further sparsening of the population code. Activated Kenyon cells were highly odor specific. Furthermore, the onset of Kenyon cell responses to projection neurons occurred within the first $200 \mathrm{~ms}$ and complex temporal patterns were transformed into brief phasic responses. Thus two types of transformations occurred within the MB: sparsening of a combinatorial code, mediated by pre- and postsynaptic processing within the mushroom body microcircuits, and temporal sharpening of postsynaptic Kenyon cell responses, probably involving a broader loop of inhibitory recurrent neurons.
\end{abstract}

\section{N T R O D U C T I O N}

The main olfactory brain regions in insects are the antennal lobes (AL) and the mushroom bodies (MB; Fig. 1A). The AL is the first synaptic processing station and a functional homologue to the vertebrate olfactory bulb (Hildebrand and Shepherd 1997). The AL of honeybees consists of $\sim 160$ subcompartments, called glomeruli (Flanagan and Mercer 1989; Galizia et al. 1999). Projection neurons (PN) convey the output of glomeruli to higher brain areas as the lateral horn and the MB (Bicker et al. 1993; Mobbs 1982). The MBs are multisensory integration centers that play a dominant role in odor learning (Davis 1993; Heisenberg 2003; Menzel 1999). They are densely packed with 170,000 Kenyon cells (KCs), which receive second-order sensory input in the $\mathrm{MB}$ calyces with different modalities innervating spatially distinct areas (Gronenberg 1986; Mobbs 1982; Schröter and Menzel 2003).

Address for reprint requests and other correspondence: P. Szyszka, Institut für Biologie-Neurobiologie, Freie Universität Berlin, Königin-Luise-Strasse 28/30, 14195 Berlin, Germany (E-mail: szyszka@zedat.fu-berlin.de).
Olfactory input is confined to the lip region. $\mathrm{KC}$ axons target output neurons in the vertical and medial lobes of the MB (also called $\alpha$ - and $\beta$ - lobes) (Kenyon 1896; Mobbs 1982; Rybak and Menzel 1993). In these areas, GABAergic feedback neurons receive input and project back to the $\mathrm{MB}$ lip region, forming an inhibitory loop (Grünewald 1999a; Schäfer and Bicker 1986).

Electrophysiological recordings in locusts and imaging experiments in Drosophila indicate that odor representations do indeed differ remarkably in the AL and the MB. Unlike PNs, $\mathrm{KC}$ s respond to odors in a sparse way (Perez-Orive et al. 2002; Stopfer et al. 2003; Wang et al. 2004). However, it is unclear whether this transformation of odor representation is a result of the KC's integration properties (as suggested for locust) or preand postsynaptic processing within the $\mathrm{MB}$ lip region. In the MB lip, the bouton-like PN terminals are involved in reciprocal and feed-forward microcircuits between GABAergic neurons and PNs (Fig. 1C) (Ganeshina and Menzel 2001). The existence of these circuits indicates that the information flow from PNs onto KCs may not only be shaped by feed-forward processes but may include interactions between PNs and GABAergic neurons within the MB microcircuits. We have therefore addressed the question of whether output activity of PN boutons is modified by presynaptic processing within the $\mathrm{MB}$ by comparing odor-evoked responses in $\mathrm{PN}$ dendrites within the $\mathrm{AL}$ glomeruli and their boutons at the $\mathrm{KC}$ synapse. To reveal transformations taking place in the postsynaptic $\mathrm{KCs}$, we recorded odor-evoked responses in $\mathrm{KCs}$ and compared them to their presynaptic input from PNs.

At all three processing stages, odors reliably evoke combinatorial activity patterns. However, in contrast to PNs, KCs code odors in a sparse way and generate only brief responses at stimulus onset. We found that $\mathrm{KC}$ 's high odor-specificity originates at two steps: first, in a presynaptic sharpening of PN synaptic output and second, in a sparsening in $\mathrm{KC}$ response. Interestingly, the temporal sharpening of $\mathrm{KCs}$ ' responses is established only at the postsynaptic side. Our results also show that PN activity generated within the first $200 \mathrm{~ms}$ determine whether a $\mathrm{KC}$ will respond.

\section{METHODS}

\section{In vivo bee preparation and dye loading}

Experiments were performed with forager bees, Apis mellifera carnica. Bees were collected at the hives, chilled, and fixed as a whole

\footnotetext{
The costs of publication of this article were defrayed in part by the payment of page charges. The article must therefore be hereby marked "advertisement" in accordance with 18 U.S.C. Section 1734 solely to indicate this fact.
} 
A
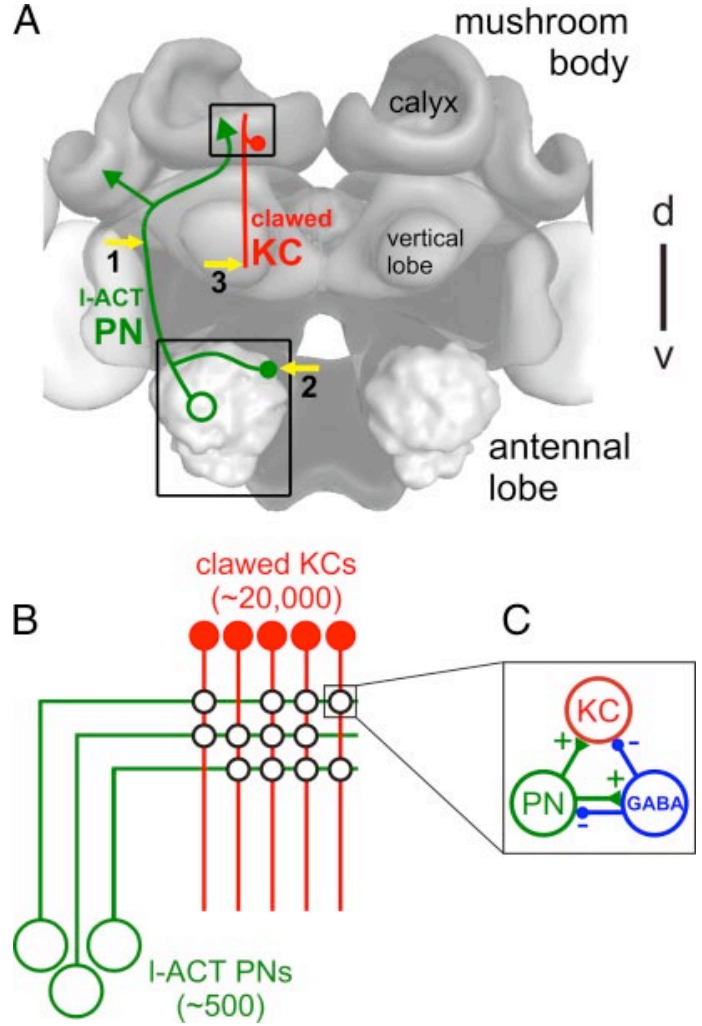

FIG. 1. The olfactory pathway in the bee brain. A: scheme of the bee brain. Lateral antennocerebralis tract projection neurons (1-ACT PN, green) were optically recorded at their dendrites in the antennal lobe (AL) and at their presynaptic boutons in the lip of the mushroom body (MB) calyx. Dendrites and somata of clawed Kenyon cells (clawed KC, red) were recorded in the MB calyx. Yellow arrows indicate sites of dye injection: 1, recording $\mathrm{PN}$ dendrites in the $\mathrm{AL} ; 2$, recording $\mathrm{PN}$ boutons in the $\mathrm{MB}$ calyces; and 3, recording clawed $\mathrm{KCs}$ in the MB calyces. Squares represent the 2 imaged areas, $\mathrm{AL}$ (for measuring PN dendrites) and MB calyx (for measuring $\mathrm{PN}$ boutons and/or clawed $\mathrm{KCs}$ ) $B$ : wiring diagram illustrates the divergent-convergent connectivity between PNs and clawed KCs. About 400 1-ACT PNs synapse onto roughly 20,000 clawed KCs. The dendrites of clawed $\mathrm{KCs}$ are small, arranged in columns, and feature few claw-like synaptic specializations (black circles). $C$ : within the lip region, PNs synapse onto GABAergic neurons which, in turn, make inhibitory synapses with PNs and KCs (adapted from Ganeshina and Menzel 2001, with permission) Moreover, GABAergic feedback neurons receive input in the MB lobes and send their axons to calyx lip region. Because they leave out the ventral vertical lobe, they presumably do not receive input from clawed KCs. Thus GABAergic neurons may provide local, $\mathrm{PN}$-driven inhibitory microcircuits within the MB calyx lip and a more global inhibitory feedback loop between the MB output and input region.

animal into recording chambers. To prevent visual stimulation through light exposure during optical recordings, the complex eyes were covered with dental wax mixed with charcoal. To allow access to the brain, the head capsule was opened by removing a piece of cuticle surrounded by the antennae, the ocelli, and the complex eyes. Where necessary, pieces of trachea covering the injection sites were removed. Neurons were stained with dextran-conjugated $\mathrm{Ca}^{2+}$ indicators by mass-injection (Fura- 2 dextran, Calcium Green-1 dextran, Molecular Probes, Eugene, OR). Injection needles were pulled from 1-mm glass capillaries to a tip diameter of $\sim 10 \mu \mathrm{m}$ and were coated with dye dissolved in $2 \%$ solution of bovine serum albumin. PN dendrites in the AL were retrogradely stained with Fura-2 dextran via their axons running through the 1-ACT, PN axon terminals were anterogradely stained with either Fura- 2 dextran or Calcium Green-1 dextran via 1-ACT PN soma clusters in the dorso-medial part of the $\mathrm{ALs}$, and dendrites and somata of clawed KCs were retrogradely stained by injecting Fura-2 dextran into the clawed KC axons located in the ventral part of the vertical lobes (see Fig. 1). Application of $\mathrm{Ca}^{2+}$ indicators with different fluorescence spectra for clawed $\mathrm{KC}$ and PN bouton staining allowed simultaneous recording of clawed KCs and PN boutons. After dye injection, the head capsule was closed with the cuticle piece and sealed with $n$-eicosan (Sigma). Half an hour later, bees were fed until satiation and kept in a container at $17-20^{\circ} \mathrm{C}$ for $8-24 \mathrm{~h}$. To prevent movement artifacts, abdomen and legs were immobilized with dental wax, muscles which innervate the antennae were carefully removed, the mouthparts were truncated and the esophagus was taken out. Immediately afterward, the head capsule was washed with bee Ringer (which contained, in mM: $130 \mathrm{NaCL}, 7 \mathrm{CaCl}_{2}, 6 \mathrm{KCl}, 2 \mathrm{MgCl}_{2}, 160$ sucrose, 25 glucose, and 10 HEPES, pH 6.7, 500 mosmol). To stabilize the brain, a $1.5 \%$ solution of low-melting agarose (Sigma, A2576) was injected into the head capsule. Experiments started $30 \mathrm{~min}$ after preparation.

\section{Odor stimulation and imaging}

We used the following odors: 1-hexanol, 2-octanol, limonene, linalool (all from Sigma), and peppermint oil (local drugstore). Odors were diluted in mineral oil to adjust for differences in vapor pressure (1-hexanol, 16.2\%; 2-octanol, 60\%; limonene, 9\%; and linalool, $46.2 \%$ ). Odor solution ( $4 \mu \mathrm{l}$ ) was applied onto a $2 \mathrm{~cm}^{2}$ piece of filter paper and placed in a plastic syringe. Using a computer controlled olfactometer (Galizia et al. 1997), odors were injected into a continuous air stream which was directed to both antennae. Odors were presented as 3-s pulses three or six times in a pseudo-randomized order with a 1-min interstimulus interval.

Images were acquired at room temperature with a sampling rate of $5 \mathrm{~Hz}$ using a TILL-Photonics imaging set up mounted on a fluorescence microscope (Olympus BX-50WI). Measurements started 2 s before stimulus onset and lasted for $12 \mathrm{~s}$. In the AL, l-ACT PN dendrites were recorded through a $\times 20$, NA $=0.95$ Olympus dip objective. Fura- 2 was excited at a single wavelength $(390 \mathrm{~nm})$ to reduce photodamage. Fluorescence was detected through a 410-nm dichroic mirror and a 440-nm long pass filter with an Imago-QE CCD camera $(1,376 \times 1,040$ pixels, $8 \times$ binned on chip to $172 \times 130)$. In the median MB calyx, KCs and PN boutons were recorded through a $\times 60,0.9$ W Olympus objective with an Imago CCD camera $(640 \times$ 480 pixels, $4 \times$ binned on chip to $160 \times 120$ ). The spatial resolution was $1.3 \mu \mathrm{m}^{2} /$ pixel and allowed resolving single 1-ACT PN boutons and clawed $\mathrm{KC}$ dendrites and somata. Fura- 2 measurements of clawed $\mathrm{KCs}$ and PN boutons were carried out with the same filter settings used for l-ACT PN dendrite recordings. For simultaneous recording of clawed KCs (Fura-2) and 1-ACT PN boutons (Calcium Green-1) the MB calyx was illuminated alternately with 390 and $475 \mathrm{~nm}$ at rate of $10 \mathrm{~Hz}$; the fluorescence was detected through a Omega 505DRLPXR dichroic mirror and a 515-nm LP filter.

\section{Data analysis}

Data were analyzed using custom-written programs in IDL (RSI, Boulder, CO). Morphological images were unsharp mask filtered in Photoshop (Adobe). $\mathrm{Ca}^{2+}$ signals were calculated as fluorescence change relative to background fluorescence $(\Delta F / F)$. Background fluorescence $(F)$ was determined by an average of five frames obtained before stimulation and was subtracted from every frame of a measurement to give $\Delta F$. Signals were corrected for dye bleaching by subtracting a logarithmic curve fitted to the mean brightness decay of the entire image frames, excluding frames during the stimulus. Because the same value was subtracted from each pixel within a frame, the bleach correction did not affect the spatial activity pattern of the $\mathrm{Ca}^{2+}$ signals (Galizia and Vetter 2004). Signals of Fura-2 have been inverted because it decreases its fluorescence at 390-nm excitation light in response to increasing $\mathrm{Ca}^{2+}$ concentrations. Activity patterns 
are depicted as color-coded images, representing the averaged $\Delta F / F$ values of 15 frames (3 s) during the odor stimulus. For better visualization a spatial low-pass filter $(3 \times 3$ pixels $)$ was applied onto the images. To analyze the response dynamics, activity patches, corresponding to responsive PN dendrites, $\mathrm{PN}$ boutons, $\mathrm{KC}$ dendrites, and $\mathrm{KC}$ somata during the 3 -s odor pulse, were selected. These pixels were averaged without filtering. The time courses obtained were then analyzed in Excel (Microsoft). For analyzing the dynamics of $\mathrm{Ca}^{2+}$ signals, three measurements were averaged. Noise was defined pixel wise as the SD of the fluorescence values over a 2-s interval (10 frames) before odor stimulus. To determine the proportion of excitatory and inhibitory responses, only signals with amplitudes $>5$ times noise were analyzed. To determine the onset of responses only, signals 7 times above noise were analyzed. The response onset was defined as signal $>3$ times noise.

Correlation analysis of spatial activity patterns was performed by computing the linear correlation between them. To determine the response profiles of $\mathrm{PN}$ dendrites, boutons and $\mathrm{KC}$ somata, three measurements were averaged. Because KCs respond within $600 \mathrm{~ms}$ after the stimulus onset (Fig. $6 B$ ), only responses occurring within the first three frames $(600 \mathrm{~ms})$ after stimulus onset were considered for PNs and KCs, to achieve comparability. Signals were considered as responses if the averaged signal during the first three frames was three times greater than noise. Isolated activity spots smaller than $2 \times 2$ pixels $\left(2.6 \mu \mathrm{m}^{2}\right)$ were regarded as noise and rejected. To detect responses of individual $\mathrm{KC}$ somata or PN boutons, scattered light was reduced by applying an unsharp mask filter (Galizia and Vetter 2004). For unsharp mask-filtering images were low-pass filtered $(30 \mu \mathrm{m}$ kernel size) and subtracted from two times the original signals. Thus images were sharpened while the absolute signal strength remained unchanged.

Due to variability in dye loading, the number and identities of stained PN boutons and KCs differed between preparations. Therefore we pooled the data coming from different animals for analyzing response profiles shown in Figs. 3 and 5. Because the probability of recording the same boutons or KCs in two different preparations was very low, we assume that pooled data reveal general properties of the 1-ACT PN boutons and clawed KCs. Although the number and identity of the PNs recorded in the AL glomeruli were similar between animals, and to make the AL data comparable to the MB data, the former were also pooled. Furthermore, within-animal analysis was performed for the AL data showing no differences to the pooled analysis (data not shown).

For evaluating how many PN boutons have to be activated for one $\mathrm{KC}$ to fire, we used binominal distribution (which in case $\mathrm{p} \rightarrow 0$ can be also approximated with Poisson distribution), set by the following formula: $P_{n}(m)=c_{n}^{m} \times p^{m}(1-p)^{n-m}$ where $c_{n}^{m}=(n !) /[m !(n-m) !]$. Final values were corrected taking into account the cumulative probability. Statistics were performed with Statistica (StatSoft.).

\section{RES ULTS}

We focused this analysis on particular populations of PNs and KCs. We exclusively recorded from uniglomerular PNs which target the lateral horn and the MB via the $1-A C T$ (Abel et al. 2001; Müller et al. 2002) and from so called clawed KCs (also termed class II or type $5 \mathrm{KCs}$ ) (Farris et al. 2004; Rybak and Menzel 1993; Strausfeld 2002). Neurons were selectively stained by injection of the membraneimpermeable $\mathrm{Ca}^{2+}$ indicators Fura-2 or Calcium Green-1 dextran into axons of 1-ACT PNs for dendrite recordings in the $\mathrm{AL}$ and axon bundles of clawed $\mathrm{KCs}$ for recordings in the $\mathrm{MB}$ lip or clusters of somata for $\mathrm{PN}$ bouton recordings in the $\mathrm{MB}$ lip (Fig. 1A). Both dyes have similar $\mathrm{Ca}^{2+}$-binding affinities and kinetics and therefore resolve the intracellular $\mathrm{Ca}^{2+}$ dynamics equally well (Konishi and Watanabe 1995; Kreitzer et al. 2000).

\section{Response properties of l-ACT PN dendrites (AL glomeruli)}

Dendritic $\mathrm{Ca}^{2+}$ signals of PNs were recorded in the AL glomeruli (Fig. 2A). Previous studies have shown that glomerular $\mathrm{Ca}^{2+}$ transients correlate with the action potential activity of PNs, which arborize in the same glomerulus (Galizia and Kimmerle 2004). Therefore we considered the glomerular $\mathrm{Ca}^{2+}$ signal of PN dendrites as a monitor for the activity of individual PNs at the output side of the glomerulus.

We measured signals from 1-ACT PNs. These neurons are uniglomerular and receive afferent input from 1 of $\sim 70$ glomeruli on the dorsorostral part of the AL (Flanagan and Mercer 1989; Galizia et al. 1999). On the average $27 \pm 0.5$ (means \pm $\mathrm{SE}$ ) of these 70 glomeruli were visible in each animal (Fig. $2 A)$. A total of 370 glomeruli from 14 bees were imaged. As previously shown (Sachse and Galizia 2002), PNs showed background activities in the absence of olfactory stimulation. Odor pulses evoked combinatorial activity patterns in PN dendrites across glomeruli (Fig. 2B). Intracellular calcium also increased in their somata. Most of the dendritic PN responses were excitatory $(98.5 \%)$, but some $(1.5 \%)$ were inhibitory. Signal size ranged from 0.5 to $8 \%(2.41 \pm 0.02 \% \Delta F / F$ of 390 nm Fura signal). Responses were phasic-tonic and/or complex, which corresponds to the temporal dynamics observed for intracellularly recorded spike rates (Abel et al. 2001; Galizia and Kimmerle 2004; Müller et al. 2002) (Fig. 2C). Of the 27 glomeruli accessible from the frontal aspect, $5.3 \pm 0.7$ were activated by any of the four odors presented, resulting in a glomerular response probability of $p_{\mathrm{G}}=0.20 \pm 0.02$ per PN and odor.

\section{Response properties of l-ACT PN boutons (MB calyx)}

To investigate the relationship between AL output and MB input carried by PNs, we next examined the response properties of PN boutons in the MB calyx. PN boutons were selectively filled with the $\mathrm{Ca}^{2+}$ indicator via l-ACT PN soma clusters located on the dorsomedial surface of the AL (Fig. 2A). We recorded from a total of 105 responsive boutons ( 9 bees) in the frontal lip area of the median calyx. Odor-induced responses consisted of isolated activity spots, which were colocalized with boutons visible in the raw fluorescence image (Fig. 2D). A total of $87.2 \%$ of the responses were excitatory, with fluorescence changes ranging from 0.3 to $3.8 \%(0.87 \pm$ $0.03 \% \Delta F / F)$, whereas the remaining $12.8 \%$ of the responses were inhibitory. As is the case in the AL, stimulation with odors activated odor-specific ensembles of PN boutons (Fig. $2 E$ ). Boutons were located at different focal depths and were not always visible in the raw fluorescence image, so we did not determine their response probability.

The response dynamics of PN boutons resembled those of PN dendrites (glomeruli). They showed phasic-tonic and/or complex time courses and often outlasted the odor pulse by several seconds (Fig. 2F). The across-odor response profile of the PN boutons also resembled the one observed in PN dendrites (Fig. 3): Limonene odor elicited fewer responses than the 
other three tested odors, and glomeruli and boutons responding to 2-octanol often also responded to 1-hexanol. However, in boutons the proportion of inhibitory responses was higher than in glomeruli (12.8 vs. $1.5 \%)$.
Response properties of clawed KC (MB calyx)

In the MB calyx, l-ACT PNs synapse onto clawed KC, a population of cells intrinsic to the MBs. To investigate how odor information is processed within the $\mathrm{MB}$, we therefore
A
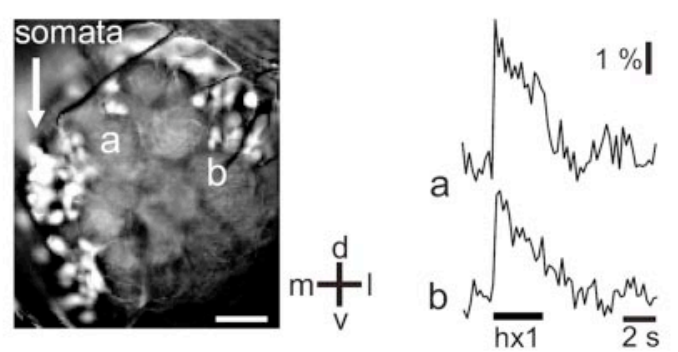

B
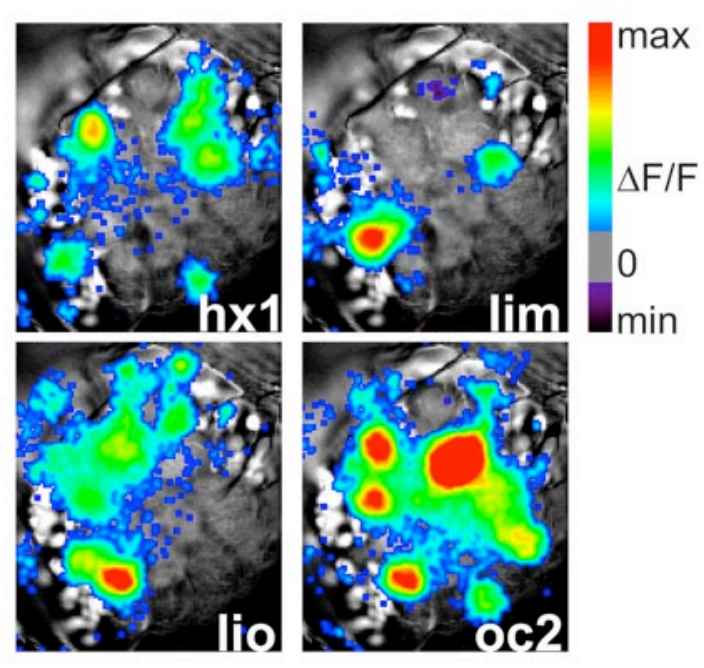

D
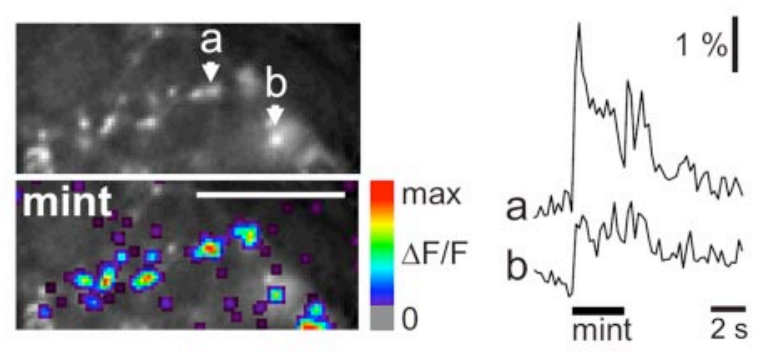

E
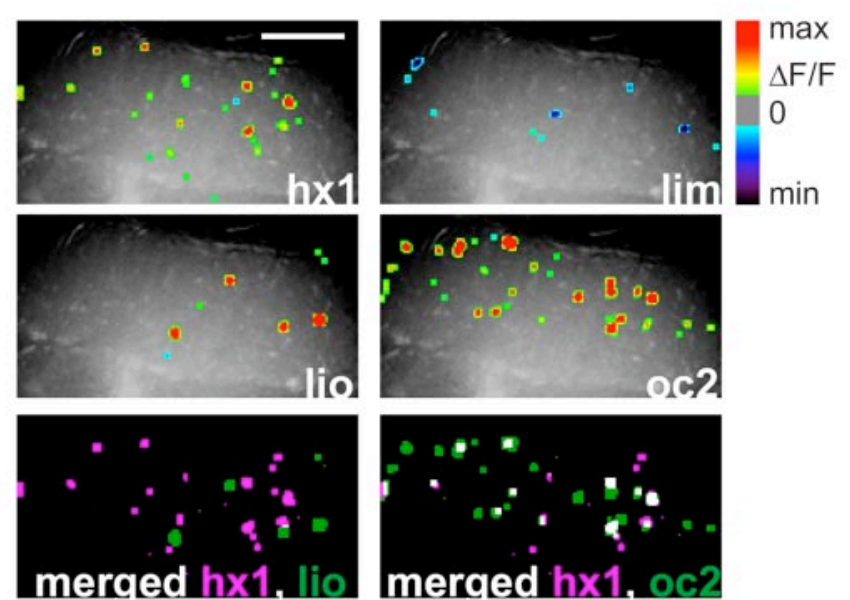

C excitation inhibition

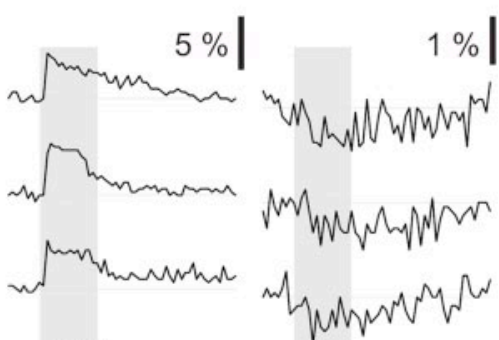

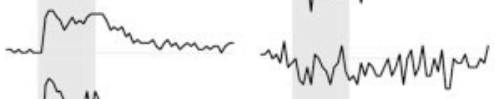

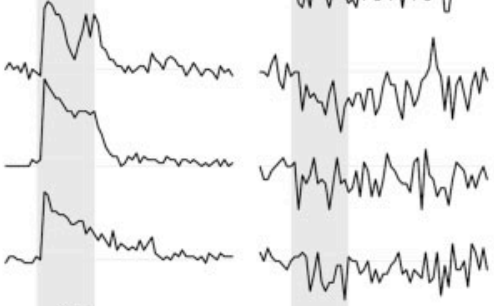

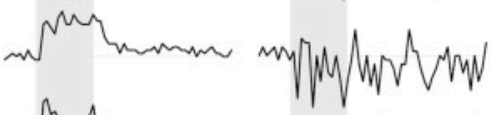

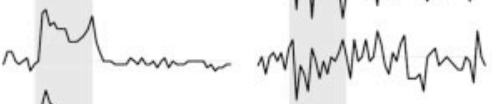

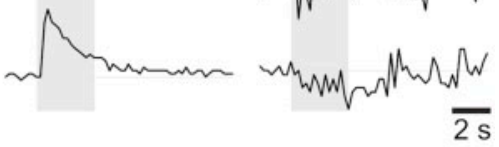

F or $1 \%$ | $1 \%$

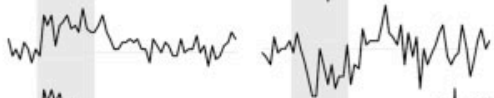

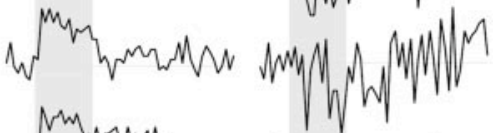

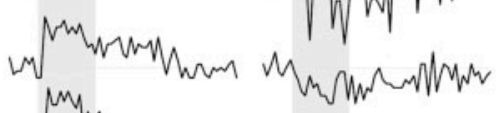

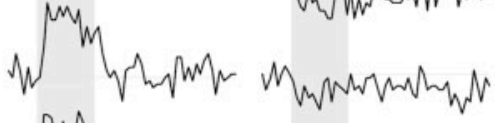

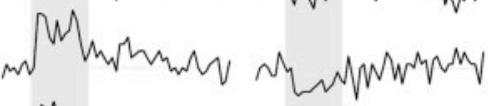

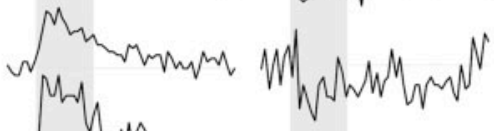

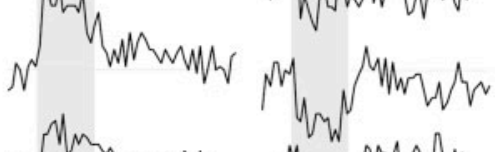

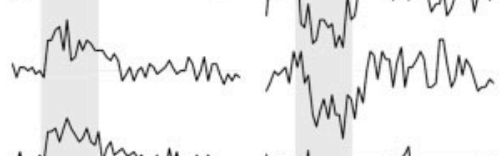

odor 


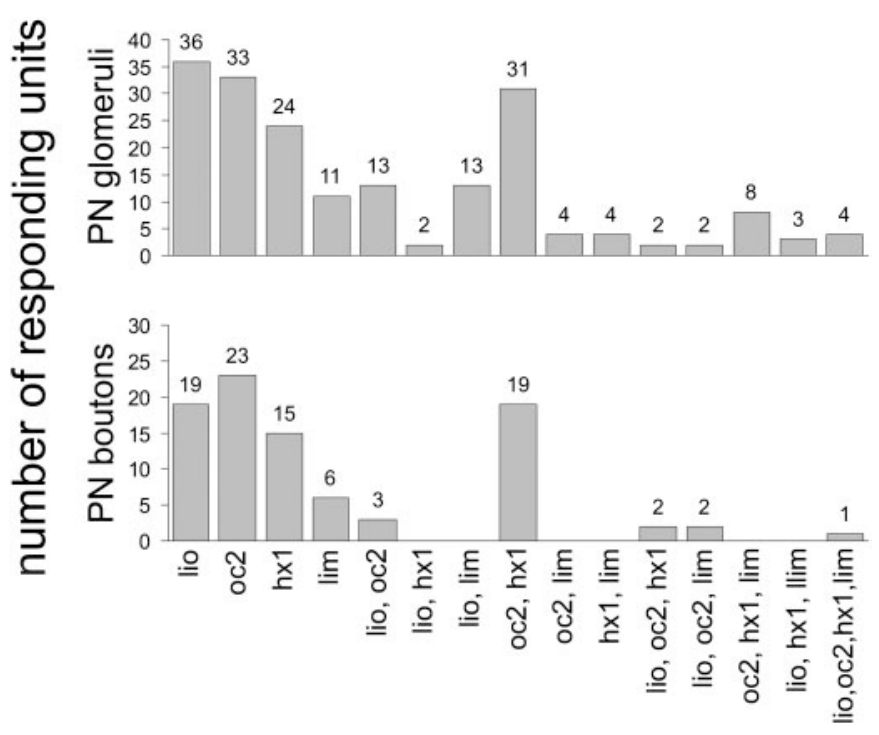

FIG. 3. Response profiles of 1-ACT PN dendrites and boutons. Histograms show the number of PN glomeruli (measured as PN dendrites) and PN boutons (measured in the calyces) that responded to either 1 or more odors. Both PN dendrites and boutons showed fewer responses to limonene (lim) than to the other three odors. 2-octanol (oc2) and 1-hexanol (hxl) activated overlapping sets of glomeruli and boutons, respectively. Boutons showed fewer overlapping responses than dendrites. (PN dendrites measured in 190 glomeruli in $n=$ 11 bees, 90 boutons in $n=8$ bees)

recorded from clawed $\mathrm{KCs}$. Clawed $\mathrm{KCs}$ can be reliably identified based on their anatomy (Fig. 4, $A$ and $B$ ). They differ from other $\mathrm{KC}$ types in that they feature a columnar arrangement of their unbranched dendrites, which possess 5-10 clawlike synaptic structures. Their somata (3-7 $\mu \mathrm{m}$ in diameter) are located outside the calyx region rostral to the lip and are connected to the dendrites through a 10 - to $100-\mu \mathrm{m}-\mathrm{long}$ primary neurite. Dendrites and somata of these cells were selectively stained by injecting Fura- 2 dextran into their axons in the ventral part of the vertical lobe. Dendritic and somatic responses to odors were then recorded in the lip region of the MB calyx. Figure $4 B$ shows an example where only one clawed $\mathrm{KC}$ was stained. Its soma and dendritic branches were visible in the fluorescence image. The time courses of $\mathrm{Ca}^{2+}$ transients in dendrite and soma were similar. Both followed a uniform phasic upraise at stimulus onset with off responses after the end of the odor stimulus. However, $\mathrm{Ca}^{2+}$ signal decay was slower in the somata.

In the soma layer, odors induced sharp activity peaks in individual clawed $\mathrm{KC}$ somata, which showed fluorescence changes ranging from 0.3 to $2.7 \%$ (1.09 $\pm 0.03 \Delta F / F, 67$ responses in 8 bees). In the lip neuropil of the calyx, odorinduced dendritic activity appeared in larger, less well-defined regions and showed fluorescence changes ranging from 0.5 to $6 \%(1.49 \pm 0.03 \% \Delta F / F, 473$ responses in 15 bees $)$. Those activity patches had a columnar or elongated shape of 20-60 $\mu \mathrm{m}$ in diameter, which matches the morphology of the dendritic trees of single-clawed KCs (Fig. 4, $B$ and $D$ ). Dendritic activity patches and activated somata often occurred close to each other, suggesting that they belong to the same clawed KC. Response dynamics were common among clawed KCs. Figure $4 C$ shows 10 representative dendriticclawed $\mathrm{KC}$ responses. Clawed $\mathrm{KC}$ exclusively exhibited excitatory responses. Off responses were visible in $40 \%$ of the measurements.

Different odors induced different patterns of activated clawed KC dendrites with different degrees of overlap, whereas stimulation with the same odor reliably activated the same clawed $\mathrm{KC}$ ensembles (Fig. 4D). To confirm the odor specificity of the spatial response patterns, the correlation coefficient between dendritic activity patterns was calculated as a measure of similarity. The correlation coefficients were generally low, something that can be attributed to the fact that only a small surface of the imaged area was activated by any one stimulus, so that correlation coefficients were strongly influenced by the noise in the nonresponding areas. The mean correlation coefficient between responses that have been elicited by repeated presentation of the same odor was always higher than between responses to different odors $(0.37 \pm 0.04$ vs. $0.14 \pm 0.04$, Wilcoxon signed-rank test, $P<0.001, n=$ 15; Fig. 4E).

Due to the limited spatial resolution of the signal, it was not possible to determine whether the overlap between the dendritic activity patches reflects responses from the same clawed $\mathrm{KC}$ or from clawed $\mathrm{KCs}$ with anatomically overlapping dendritic trees. However, we found that the responses in somata mirrored the activity of their corresponding dendrites. Therefore we quantified the response properties of individual clawed $\mathrm{KCs}$ by analyzing the activity of somata as a monitor for individual KCs. We recorded from 1680 clawed $\mathrm{KC}$ somata in 12 animals, which were exposed to all four odors. Seventyseven somata responded to at least one of the four tested odors. Odors activated nonoverlapping clawed $\mathrm{KC}$ ensembles. The response probability of a given clawed $\mathrm{KC}$ was $p_{\mathrm{KC}}=0.013 \pm$ 0.001 (means $\pm \mathrm{SE}$ ) per odor.

\section{Sparsening of the population code in the $M B$}

To analyze the transformation of odor representations in the $\mathrm{MB}$ and to evaluate the contribution of pre- and postsynaptic processing, we compared the responses of PN dendrites, PN boutons, and clawed KCs to the four tested odors (1-hexanol, 2-octanol, limonene, and linalool). We first asked whether odor processing along the olfactory pathway leads to a change in the sparseness of the population code. The term "sparseness" describes the proportion of active units at any time (population sparseness) and/or the mean tuning width of each neuron

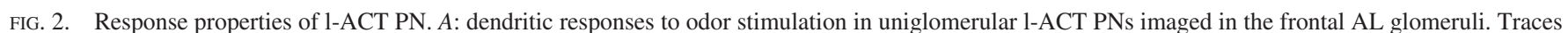

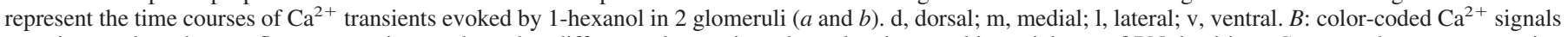

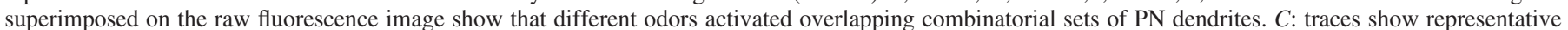

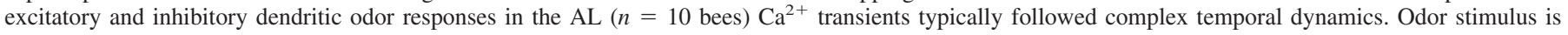

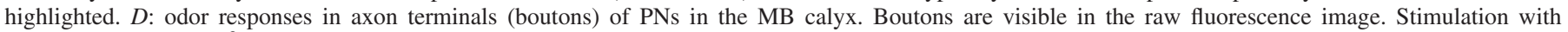

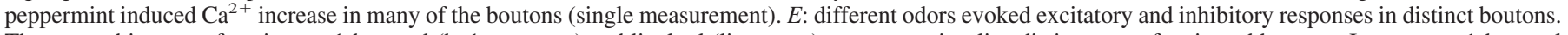

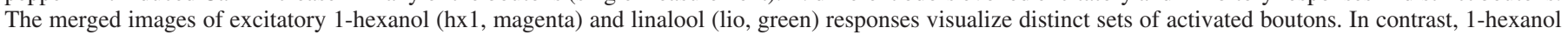

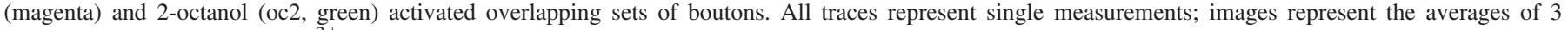

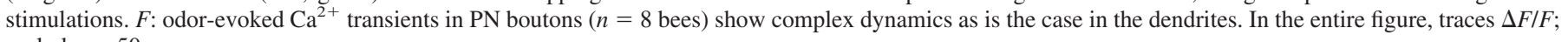
scale bars: $50 \mu \mathrm{m}$ 


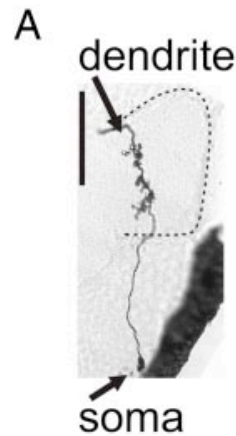

B
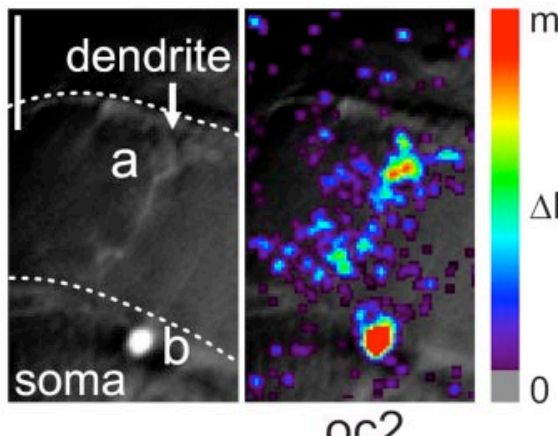

$\max$

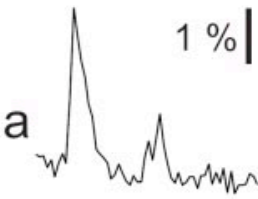

C
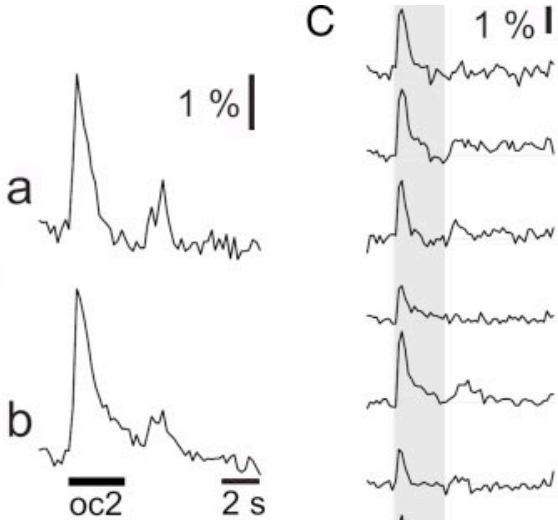

oc2

D

lio

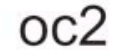

0.16

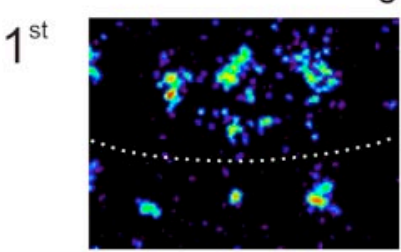

0.42

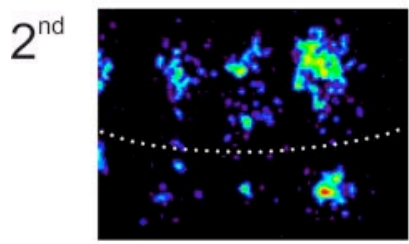

0.19

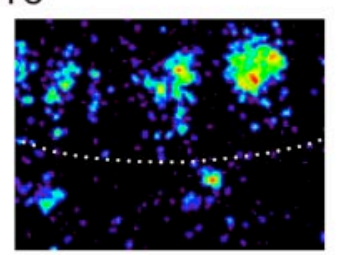

0.56

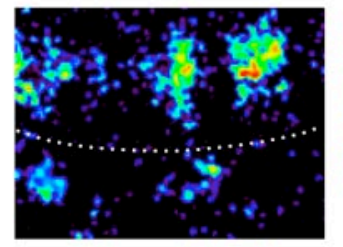

$$
\text { (1) }
$$

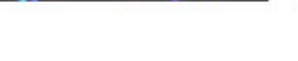

\section{$\mathrm{mer}$}

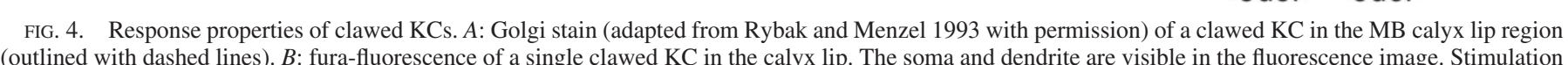

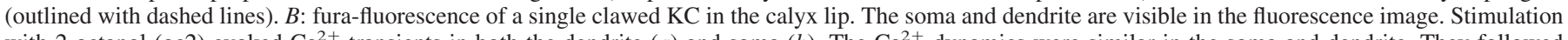

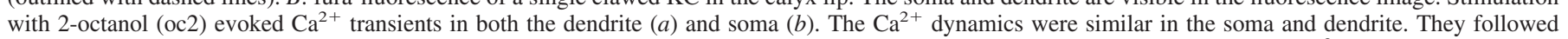

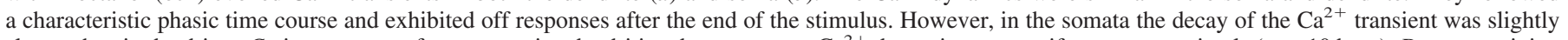

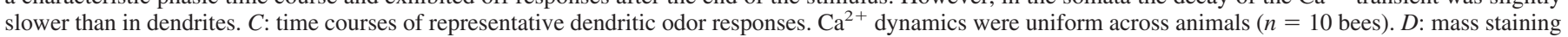

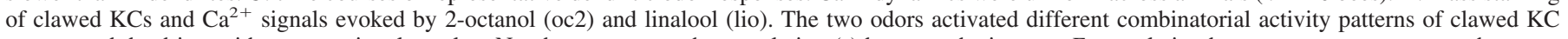

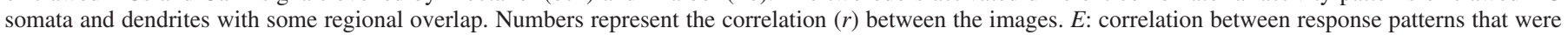

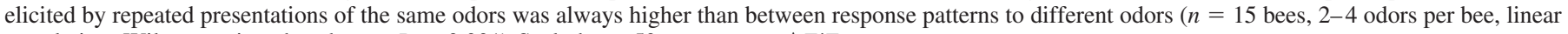
correlation, Wilcoxon signed-rank test, $P<0.001)$ Scale bars: $50 \mu \mathrm{m}$; traces: $\Delta F / F$.

(lifetime sparseness). A sparse code is characterized by few neurons active at any time and/or a narrow tuning width (Olshausen and Field 2004; Willmore and Tolhurst 2001). Figure 5 illustrates the progressive sparsening of the odor code from PN dendrites to PN boutons and to clawed $\mathrm{KC}$ somata. In the AL, PNs were broadly tuned because many glomeruli respond to more than one odor. PN boutons were more narrowly tuned, as the histogram was skewed toward a lower number of odors. Clawed KCs showed the highest sparseness, were extremely odor specific, and responded mostly to one odor only. This result demonstrates that odor processing in the $\mathrm{MB}$ results in a sparsening of the odor code carried by clawed KCs. Furthermore it indicates that sparsening of the population code occurs not only at the level of the postsynaptic clawed KCs but, to a lower extent, also at the presynaptic terminals of the PNs. Thus the high degree of sparseness at the $\mathrm{KC}$ level can be attributed to at least two distinct processes.

\section{Change in response dynamics}

Understanding the logic of sensory information coding requires the identification of those features of the neural representations that are read out by downstream neurons. We therefore tried to narrow down features of the PN responses that might be read out by clawed KCs. Odor-evoked activity patterns of PNs are not stationary, but show temporal modulations, which evolve over time and lead to activity patterns that in several species are most odor-specific after some hundreds of milliseconds following odor onset (bee: Galán et al. 2004; Galizia et al. 2000; locust: Stopfer et al. 2003; zebrafish olfactory bulb: Friedrich and Laurent 2001). It has been suggested that downstream neurons have to read out these temporal patterns to retrieve the odor information. If that is true, then the temporal patterns of $\mathrm{PN}$ responses must be relevant for $\mathrm{KCs}$, and $\mathrm{KCs}$ tuned to $\mathrm{PN}$ activity patterns should respond with time lags corresponding to particular phases of the dynamic response. 


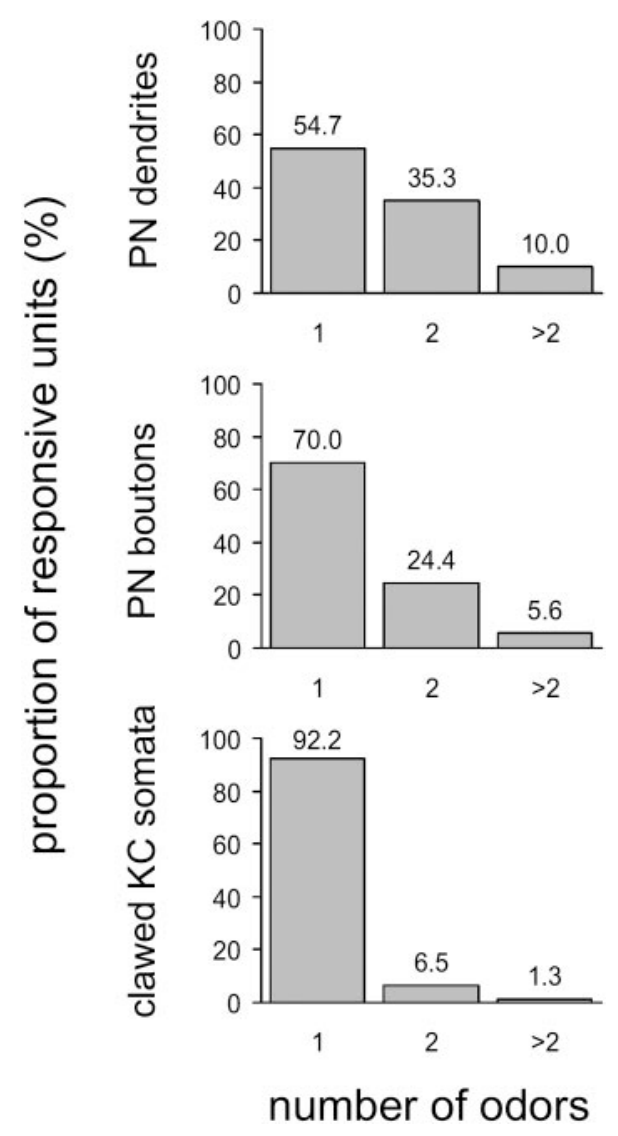

FIG. 5. Progressive sparsening of population responses in the MB. The histograms show the percentage of responsive units (PN dendrites measured in the glomeruli, PN boutons, and clawed KC somata) that were activated by 1 , 2 , or more odors ( 4 odors were presented) The distributions varied significantly between the different processing stages and showed a progressive increase in the sparseness of odor representations from PN dendrites to PN boutons and to clawed KCs $\left(\chi^{2}\right.$ test, applied on absolute numbers of responsive units, $P<$ 0.001; PN dendrites: $n=298$ responsive glomeruli in 14 bees; PN boutons: 90 responsive in 9 bees; clawed KCs: 77 responsive somata in 12 bees)

To test this hypothesis, we investigated the temporal relationship between PN and clawed $\mathrm{KC}$ responses by simultaneously recording from $\mathrm{PN}$ boutons and clawed $\mathrm{KC}$ using two different dyes. The traces in Fig. $6 A$ show $\mathrm{Ca}^{2+}$ transients measured at $5 \mathrm{~Hz}$ in individual PN boutons and colocalized $\mathrm{KC}$ dendrites. There was no detectable time lag between the responses of $\mathrm{PN}$ boutons and clawed $\mathrm{KCs}$, indicating that the delay of transmission was well below the $200-\mathrm{ms}$ cycle duration in the recordings. To get a more accurate estimation of the delay between PN boutons and $\mathrm{KC}$ responses, we compared response onsets to 3-s odor pulses. Only animals with signal/ noise ratios $>5$ were chosen, and three measurements were averaged (PNs: 3 times 655 measurements in 11 bees; KCs: 3 times 184 measurements in 15 bees).

Most of the PNs responded with constant delays. $90 \%$ of the responses occurred within $400 \mathrm{~ms}$ after stimulus onset, whereas the remaining $10 \%$ showed delays of $\leq 1,000 \mathrm{~ms}$. Ninety-five percent of the KC responses occurred within 400 $\mathrm{ms}$ after stimulus onset, and the remaining $5 \%$ occurred within $400-600 \mathrm{~ms}$. No KC responded at a later time point. Figure $6 B$ shows the time courses of $\mathrm{Ca}^{2+}$ signals in PN dendrites, boutons, and $\mathrm{KCs}$ and illustrates the relationship between the response dynamics of PNs and KCs. Even though PN activity was temporally complex during an odor stimulus of $3 \mathrm{~s}$ and often outlasted the odor stimulus, responses in KCs were always limited to brief phasic responses. These results show that the onset of KCs responses occurs within $<200 \mathrm{~ms}$ of the initial PN responses, whereas later input does not activate KCs. The results also demonstrate that the temporal sharpening of the $\mathrm{KC}$ response reflects postsynaptic processing because it is not present in PN boutons.

Figure 7 elucidates our findings and illustrates a proposed mechanism that may mediate the transformation of odor representations along the olfactory path (see DISCUSSION): Within the AL, odors evoke dense across-fiber patterns of l-ACT PNs. The spatial activity pattern of the presynaptic terminals of these projection neurons is sparsened within the MB lip-presumably through reciprocal microcircuits between GABAergic neurons and 1-ACT PNs. Clawed KCs integrate 1-ACT PN activity within $200 \mathrm{~ms}$ and transform the complex temporal pattern into brief phasic responses. The sharpening of clawed KCs' temporal responses may be mediated by a more global inhibition via GABAergic feedback neurons. Moreover the PN-to-KC divergence together with a high spiking threshold may lead to a further sparsening of clawed $\mathrm{KC}$ population responses.

\section{I S C U S S I O N}

We characterized the transmission of odor-evoked activities along three consecutive processing stages and analyzed preand postsynaptic mechanisms that underlay odor processing in the honeybee MBs. We found that odor coding in the AL and the MBs are fundamentally different. The representation of odors in the MBs is sparse across the population of clawed KC, and this sparsening occurs in two separate steps, one presynaptic and the other postsynaptic at the PN-KC synapse. Furthermore, odor representation in clawed KCs is temporally sharp, indicating that at most the initial $200 \mathrm{~ms}$ of an odor response are relevant for clawed $\mathrm{KC}$ responses. This has important implications for how information can be used for olfactory recognition and memory formation.

\section{Methodological aspects}

To investigate the spatial and temporal properties of odor processing in the $\mathrm{MB}$, we measured $\mathrm{Ca}^{2+}$ transients in 1-ACT $\mathrm{PN}$ dendrites in the AL, in their presynaptic axon terminals in the MB, and in their postsynaptic partners, the clawed KCs. The measured $\mathrm{Ca}^{2+}$ transients in the three sub-structures are most likely dominated by calcium influx through voltageactivated calcium channels and qualitatively reflect the electrical activity of their respective cell compartment. For PN dendrites, intracellular recordings and subsequent $\mathrm{Ca}^{2+}$ imaging of the corresponding glomerulus have shown that $\mathrm{Ca}^{2+}$ transients are directly related to their action potential rates, i.e., PNs that responded to an odor with increased spiking also responded with an increase in dendritic $\mathrm{Ca}^{2+}$ (Galizia and Kimmerle 2004). Studies in many neurons from a variety of species showed that pre- and postsynaptic $\mathrm{Ca}^{2+}$ transients measured with Fura-2 or Calcium Green-1 are dominated by $\mathrm{Ca}^{2+}$ influx through voltage-gated channels (Charpak et al. 2001; Kurtz et al. 2001; Oertner et al. 1999; Single and Borst 2002; Svoboda et al. 1997; Wachman et al. 2004). 
A
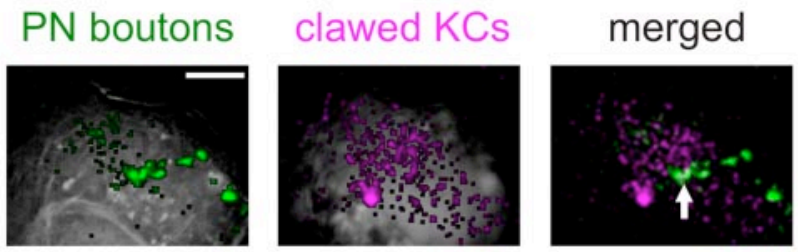

bee 1
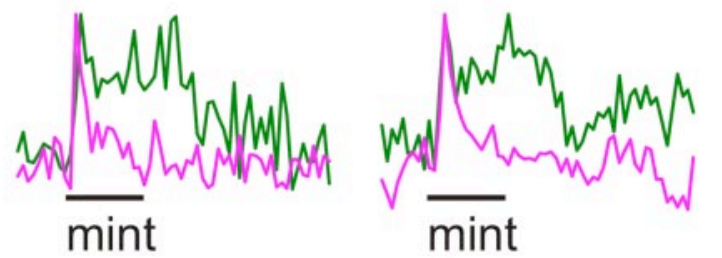

bee 2
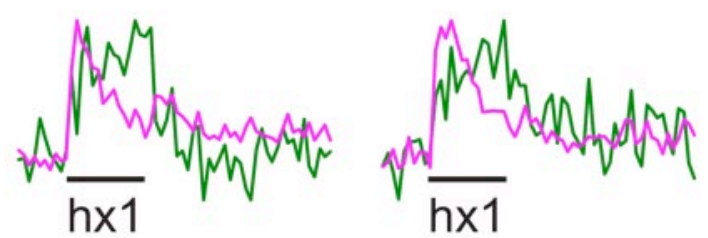

bee 3
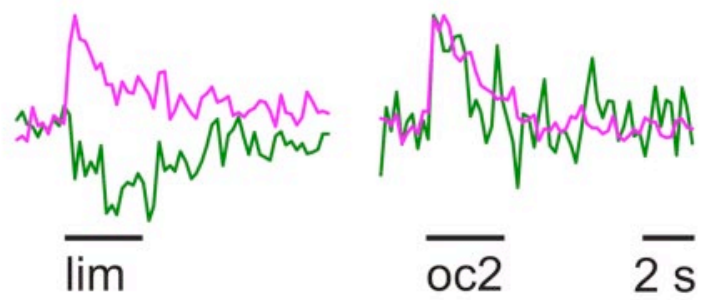

B
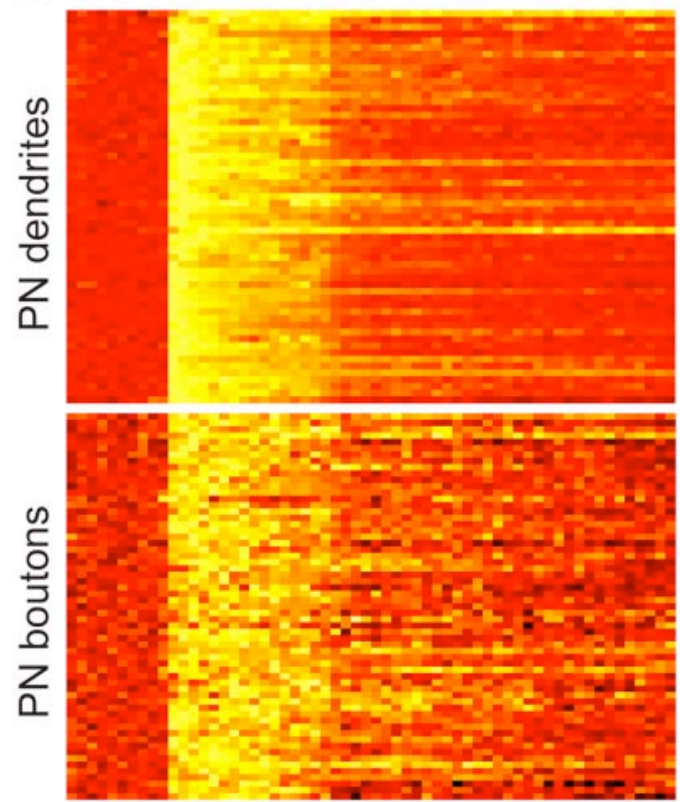

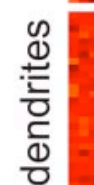

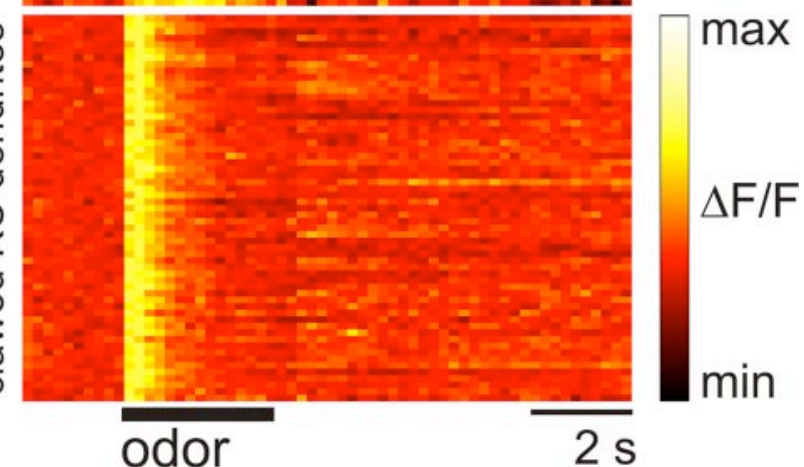

FIG. 6. Sharpening of response dynamics in the MB. A: 1-ACT PN boutons and clawed KCs were stained with different $\mathrm{Ca}^{2+}$ indicators $\left(\mathrm{PNs}^{2} \mathrm{Calcium}\right.$ Green-1, KCs: Fura-2), and their odor responses were simultaneously recorded in the MB calyx lip. The merged images display overlapping PN bouton and clawed KC activities. The traces represent responses in a PN bouton and in clawed KC dendrites located in the same area (arrow) and for 2 further bees. The $\mathrm{Ca}^{2+}$ transient of the PN bouton responses typically followed a tonic time course, whereas the clawed KC dendrites showed a phasic dynamic. No time lag was observed between PN and clawed KC bouton responses. Note the off response of the dendritic KC signal coinciding with the end of the PN responses in bee 2. $B$ : in each block, the time courses of 60 individual glomeruli (PN dendrites), PN boutons, or KC dendrites are depicted, each recorded for $12 \mathrm{~s}$. The odor stimulus is marked with the black bar. The signal intensity is color-coded. Clawed KCs transformed the long-lasting and complex PN input into brief responses. Traces were normalized to the maximum of the absolute signal amplitude. Images and traces represent single measurements. Scale bar $50 \mu \mathrm{m}$; traces $\Delta F / F$ normalized to maximum

We confined the area monitored in the MB to the frontal part of the lip for technical reasons. We assume that the properties of PN boutons and clawed KCs located in this area are representative for any other part of the MB lip. Within the MB calyx, KCs of different types and sensory modalities are arranged in concentric layers. Olfactory KCs are homogenously distributed along the lip layer, where they receive input from the 1-ACT PN boutons (Mobbs 1982). Although 1-ACT PN boutons are not homogenously distributed (Müller et al. 2002), the recorded area was large enough to contain several boutons of each PN.

Besides l-ACT PNs recorded in this study, m-ACT PNs also project onto the lip area. $\mathrm{m}$-ACT PNs receive input from an area in the AL that was not accessible for our imaging technique. Müller et al. (2002) found that $\mathrm{m}$ - and l-ACT PNs differ in their response properties. Unlike 1-ACT PNs, m-ACT PNs show odor-specific response latencies of several $100 \mathrm{~ms}$. Because clawed KCs exhibit stable and immediate responses, we conclude that the recorded responses of clawed KCs are exclusively driven by 1-ACT PNs. In the same area where we recorded from clawed KCs, there are other KCs (Type $1 \mathrm{KCs}$ ) that might be the preferential targets for m-ACT PNs. It is likely that such KCs receiving input from $\mathrm{m}$-ACT PNs have very different response properties from the clawed KCs described here, but these KCs were not filled with dye and did not contribute to the recorded signal.

\section{Transformation of odor coding within l-ACT PNs: dendrites to boutons}

Within the MB lip, PN boutons convey excitatory input to KCs and GABAergic neurons, which in turn provide inhibitory feedback onto the PN boutons themselves (Fig. 1B) (Ganeshina and Menzel 2001). To asses the role of these microcircuits for odor processing, we compared the odor responses in PN dendrites within the AL glomeruli with those at the PN boutons 

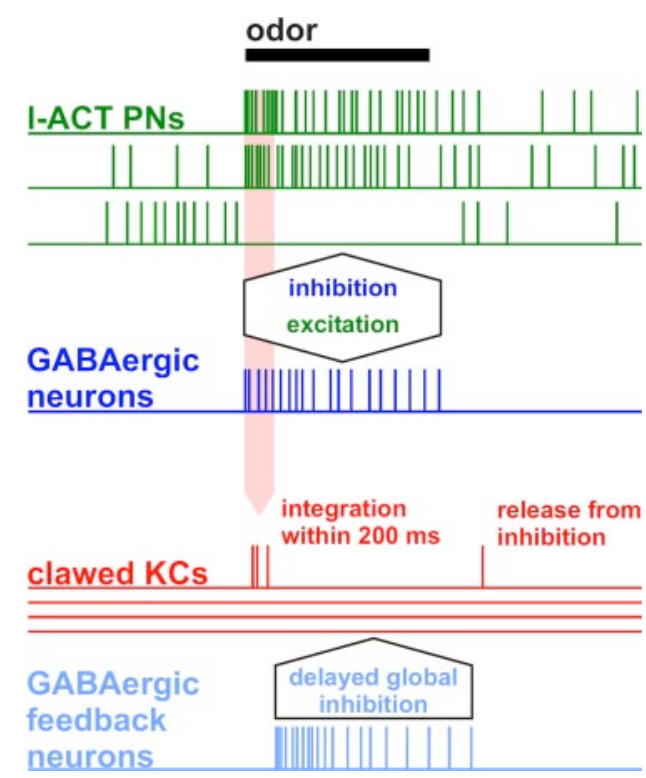

FIG. 7. Model of odor processing in the MB input. 1-ACT PNs (green) convey odor information in form of dense across-fiber patterns of action potential bursts. In the MB input, they make excitatory synapses with clawed KCs (red) and with terminals of extrinsic GABAergic neurons (dark blue), which, in turn, make inhibitory synapses with PN boutons. The inhibitory input onto PN boutons has the effect that not all boutons of an excited PN will respond, resulting in a sharpening of the response profiles of PN boutons. GABAergic feedback neurons (light blue), which receive input from a large number of KCs (presumably excluding clawed $\mathrm{KCs}$ ), form a global inhibitory loop, and mediate delayed, odor-driven inhibition of clawed KCs. The inhibitory input onto clawed KCs restricts their integration time window for PN input to $<200 \mathrm{~ms}$ and shortens their response duration. After the end of the odor stimulus, the inhibitory input declines and clawed KCs are released from inhibition and become excitable again. Because many PNs continue spiking after the end of the odor stimulus, some clawed KCs exhibit off responses. The divergent connectivity between 1-ACT PNs and clawed KCs, together with a high spiking threshold of clawed KCs, leads to a further sparsening of clawed KCs' population response.

in the MBs (Figs. 2 and 3). Both PN dendrites and boutons exhibited reliable combinatorial patterns of odor-evoked activity and showed similar response profiles. However, PN dendrites responded less frequently with inhibition than their boutons (1.5 vs. $12.8 \%)$ and were more broadly tuned to odors. We propose that this effect is caused by local inhibition of the presynaptic boutons within the MB lip. The high odor specificity observed in KCs may therefore be generated, in part, by presynaptic inhibition of PN boutons via local recurrent microcircuits.

\section{Transformation from l-ACT PNs to clawed KCs}

SPARSENING. We have analyzed the response characteristics of l-ACT PNs and clawed KCs across their population. In both, odors are coded in form of combinatorial activity patterns. However, clawed KCs with the same olfactory profile are not grouped into functional subunits as is the case for the PNs in the AL, where they all coalesce in one glomerulus. We demonstrated that odor-activated clawed $\mathrm{KC}$ ensembles are sparse compared with PN ensembles. Unlike PNs, clawed KCs responded very selectively to odors. The observed response probability $p_{\mathrm{KC}}$ of a given clawed $\mathrm{KC}$ averages 0.013 per odor and is significantly lower than the response probability of PN dendrites $\left(p_{\mathrm{G}}=0.2\right)$. The observed sparseness of the recorded clawed KCs is in accordance with the finding of a sparse code in KCs of locusts and Drosophila (Perez-Orive et al. 2002; Wang et al. 2004) and may therefore represent a general property of KCs. Both the distributive character and the sparseness of the clawed $\mathrm{KC}$ code may be a result of the divergentconvergent connectivity between PNs and clawed KCs (Laurent 2002). About 500 1-ACT PNs converge onto estimated 20,000 clawed KCs (counted and interpolated from Golgi stains, J. Rybak, personal communication). Knowing that each l-ACT PN has $\sim 400$ boutons (Müller et al. 2002), this yields a ratio of $\sim 10$ boutons per clawed KC. This matches the number of claw-like dendritic specializations in each clawed KC. Assuming that each of these claws contacts a different PN, how many of these PN inputs must be coactive to induce a clawed $\mathrm{KC}$ response? With a response probability $p_{\mathrm{G}}=0.2$ in PNs, a cumulative probability corresponding to clawed $\mathrm{KC}$ activity of $p_{\mathrm{KC}}=0.013$ is reached when 5.8 or more of $10 \mathrm{PNs}$ are simultaneously active. Thus a single clawed $\mathrm{KC}$ would compress information carried by a subset of 6-10 PNs. This means that clawed KCs must have a high firing threshold, which could be the result of the intrinsic properties of KCs and/or of odor-driven inhibition (Laurent and Naraghi 1994; Perez-Orive et al. 2002).

TEMPORAL SHARPENING. Clawed KCs had a phasic response with a fast onset of $<200 \mathrm{~ms}$. This observation conflicts with studies in locusts. There odors evoke transient spike synchronizations between PNs which develop over hundreds of milliseconds after stimulus onset (Laurent and Davidowitz 1994; Laurent et al. 1996; Stopfer et al. 2003; Wehr and Laurent 1996) and lead to KCs that respond with variable, odor-specific latencies (Perez-Orive et al. 2002; Stopfer et al. 2003). The short and stable time-lag between responses in 1-ACT PNs and clawed KCs in the bee suggests that clawed KCs decode afferent PN activities only at the beginning of each response bout. However, it cannot be excluded that action potentials that might be generated by clawed KCs after the $200 \mathrm{~ms}$ may contain information that we could not measure with $\mathrm{Ca}^{2+}$ imaging.

What are the mechanisms underlying the short integration time and the sharp response dynamics of clawed KCs? We assume that both result from odor-driven delayed inhibition of clawed KCs. Odor-driven inhibitory input was found in locust KCs (Laurent and Naraghi 1994; Perez-Orive et al. 2002). The response dynamics of bees' clawed KCs provide clear evidence that they might receive inhibitory input as well. Despite continuous input from PNs, clawed KCs' response onset was restricted to the beginning of PNs' response and followed a phasic time course (Fig. 6B). There were no clawed $\mathrm{KCs}$ that responded at a later time point. This indicates that clawed KCs might indeed receive delayed odor-driven inhibition that lasts as long as the odor stimulus and prevents them from spiking again. Candidate neurons for the presumed odor-driven delayed inhibition of $\mathrm{KCs}$ are GABAergic feedback neurons, which receive input from a large population of $\mathrm{KCs}$ in the $\mathrm{MB}$ pedunculus and lobes, presumably leaving out the clawed $\mathrm{KCs}$, and project back to the lip of the MB (Grünewald 1999a; Schäfer and Bicker 1986). These neurons exhibit phasic-tonic odor responses that usually do not exceed stimulus duration (Grünewald 1999b). Thus the off responses of clawed KCs 
after the stimulus offset could reflect release from inhibition that makes them once again sensitive for input from PNs that remains active after stimulus offset.

Clawed KCs of the $\gamma$-lobe in Drosophila are particularly important for olfactory short-term memory (Zars et al. 2000). Anatomical similarities between clawed $\mathrm{KCs}$ in bees and Drosophila suggest they are homologues (Strausfeld 2002). Accordingly, the clawed $\mathrm{KCs}$ recorded in our study might play a major role in memory formation. Indeed the code used clawed KCs might be ideally suited for computations underlying odor learning. Each odor is encoded in a quasi-simultaneous combinatorial pattern of active clawed KCs without complex temporal activity sequences, which is an ideal substrate for associative learning rules of a Hebbian type (Lechner and Byrne 1998).

\section{A C K N O W L E D G MENTS}

We are grateful to J. Rybak and A. F. Silbering for helpful discussions and M. Wurm for correcting the manuscript.

Present address of C. G. Galizia: Universität Konstanz, D-78457 Konstanz, Germany.

\section{G R A N T S}

This work was supported by grants from the Deutsche Forschungsgemeinschaft (SFB 515).

\section{REFERENCES}

Abel R, Rybak J, and Menzel R. Structure and response patterns of olfactory interneurons in the honeybee, Apis mellifera. J Comp Neurol 437: 363-383, 2001.

Bicker G, Kreissl S, and Hofbauer A. Monoclonal antibody labels olfactory and visual pathways in Drosophila and Apis brains. J Comp Neurol 335: 413-424, 1993.

Charpak S, Mertz J, Beaurepaire E, Moreaux L, and Delaney K. Odorevoked calcium signals in dendrites of rat mitral cells. Proc Natl Acad Sci USA 98: 1230-1234, 2001.

Davis RL. Mushroom bodies and Drosophila learning. Neuron 11: 1-14, 1993

Farris SM, Abrams AI, and Strausfeld NJ. Development and morphology of Class II Kenyon cells in the mushroom bodies of the honey bee, Apis mellifera. J Comp Neurol 474: 325-339, 2004.

Flanagan D and Mercer AR. An atlas and 3-D reconstruction of the antennal lobes in the worker honey bee, Apis mellifera L. (Hymenoptera: Apidae) Int J Insect Morphol Embryol 18: 145-159, 1989.

Friedrich RW and Laurent GJ. Dynamic optimization of odor representations by slow temporal patterning of mitral cell activity. Science 291: 889-894, 2001.

Galán RF, Sachse S, Galizia CG, and Herz AVM. Odor-driven attractor dynamics in the antennal lobe allow for simple and rapid olfactory pattern classification. Neural Comput 16: 999-1012, 2004.

Galizia CG, Joerges J, Küttner A, Faber T, and Menzel R. A semi-in-vivo preparation for optical recording of the insect brain. J Neurosci Methods 76 61-69, 1997.

Galizia CG and Kimmerle B. Physiological and morphological characterization of honeybee olfactory neurons combining electrophysiology, calcium imaging and confocal microscopy. J Comp Physiol 190: 21-38, 2004.

Galizia CG, Küttner A, Joerges J, and Menzel R. Odour representation in honeybee olfactory glomeruli shows slow temporal dynamics: an optical recording study using a voltage-sensitive dye. J Insect Physiol 46: 877-886, 2000.

Galizia CG, McIlwrath SL, and Menzel R. A digital three-dimensional atlas of the honeybee antennal lobe based on optical sections acquired by confocal microscopy. Cell Tissue Res 295: 383-394, 1999.

Galizia $\mathbf{C}$ and Vetter R. Optical methods for analyzing odor-evoked activity in the insect brain. In: Advances in Insect Sensory Neuroscience, edited by Christensen T. Boca Raton, FL: CRC, 2004.
Ganeshina OT and Menzel R. GABA-immunoreactive neurons in the mushroom bodies of the honeybee: an electron microscopic study. J Comp Neurol 437: 335-349, 2001.

Gronenberg W. Physiological and anatomical properties of optical inputfibres to the mushroom body in the bee brain. J Insect Physiol 32: 695-704, 1986.

Grünewald B. Morphology of feedback neurons in the mushroom body of the honeybee, Apis mellifera. J Comp Neurol 404: 114-126, 1999a.

Grünewald B. Physiological properties and response modulations of mushroom body feedback neurons during olfactory learning in the honeybee, Apis mellifera. J Comp Physiol 185: 565-576, 1999b.

Heisenberg M. Mushroom body memoir: from maps to models. Nat Rev Neurosci 4: 266-275, 2003.

Hildebrand JG and Shepherd GM. Mechanisms of olfactory discrimination: converging evidence for common principles across phyla. Annu Rev Neurosci 20: 595-631, 1997.

Kenyon FC. The brain of the bee-a preliminary contribution to the morphology of the nervous system of the Arthropoda. J Comp Neurol 6: 134-210, 1896.

Konishi $\mathrm{M}$ and Watanabe M. Resting cytoplasmic free $\mathrm{Ca}^{2+}$ concentration in frog skeletal muscle measured with fura-2 conjugated to high molecular weight dextran. J Gen Physiol 106: 1123-1150, 1995.

Kreitzer AC, Gee KR, Archer EA, and Regehr WG. Monitoring presynaptic calcium dynamics in projection fibers by in vivo loading of a novel calcium indicator. Neuron 27: 25-32, 2000.

Kurtz R, Warzecha AK, and Egelhaaf M. Transfer of visual motion information via graded synapses operates linearly in the natural activity range. J Neurosci 21: 6957-6966, 2001.

Laurent G. Olfactory network dynamics and the coding of multidimensional signals. Nat Rev Neurosci 3: 884-895, 2002.

Laurent GJ and Davidowitz H. Encoding of olfactory information with oscillating neural assemblies. Science 265: 1872-1875, 1994.

Laurent GJ and Naraghi M. Odorant-induced oscillations in the mushroom bodies of the locust. J Neurosci 14: 2993-3004, 1994.

Laurent GJ, Wehr M, and Davidowitz H. Temporal representations of odors in an olfactory network. J Neurosci 16: 3837-3847, 1996.

Lechner HA and Byrne JH. New perspectives on classical conditioning: a synthesis of Hebbian and non-Hebbian mechanisms. Neuron 20: 355-358, 1998.

Menzel R. Memory dynamics in the honeybee. J Comp Physiol 185: 323-340, 1999.

Mobbs PG. The brain of the honeybee Apis mellifera. I. The connections and spatial organization of the mushroom bodies. Phil Trans R Soc Lond B Biol Sci 298: 309-354, 1982.

Müller D, Abel R, Brandt R, Zöckler M, and Menzel R. Differential parallel processing of olfactory information in the honeybee, Apis mellifera $L$. J Comp Physiol 188: 359-370, 2002.

Oertner TG, Single S, and Borst A. Separation of voltage- and ligand-gated calcium influx in locust neurons by optical imaging. Neurosci Lett 274 95-98, 1999.

Olshausen BA and Field DJ. Sparse coding of sensory inputs. Curr Opin Neurobiol 14: 481-487, 2004.

Perez-Orive J, Mazor O, Turner GC, Cassenaer S, Wilson RI, and Laurent G. Oscillations and sparsening of odor representations in the mushroom body. Science 297: 359-365, 2002.

Rybak J and Menzel R. Anatomy of the mushroom bodies in the honey bee brain: the neuronal connections of the alpha-lobe. J Comp Neurol 334: 444-465, 1993.

Sachse S and Galizia CG. The role of inhibition for temporal and spatial odor representation in olfactory output neurons: a calcium imaging study. $\mathrm{J} \mathrm{Neu}$ rophysiol 87: 1106-1117, 2002.

Schäfer S and Bicker G. Distribution of GABA-like immunoreactivity in the brain of the honeybee. J Comp Neurol 246: 287-300, 1986.

Schröter U and Menzel R. A New Ascending sensory tract to the calyces of the honeybee mushroom body, the subesophageal-calycal tract. J Comp Neurol 465: 168-178, 2003.

Single $\mathbf{S}$ and Borst A. Different mechanisms of calcium entry within different dendritic compartments. J Neurophysiol 87: 1616-1624, 2002.

Stopfer M, Jayaraman V, and Laurent G. Intensity versus identity coding in an olfactory system. Neuron 39: 991-1004, 2003.

Strausfeld NJ. Organization of the honey bee mushroom body: representation of the calyx within the vertical and gamma lobes. J Comp Neurol 450: 4-33, 2002 . 
Svoboda K, Denk W, Kleinfeld D, and Tank DW. In vivo dendritic calcium dynamics in neocortical pyramidal neurons. Nature 385: 161$165,1997$.

Wachman ES, Poage RE, Stiles JR, Farkas DL, and Meriney SD. Spatial distribution of calcium entry evoked by single action potentials within the presynaptic active zone. J Neurosci 24: 2877-2885, 2004.

Wang Y, Guo HF, Pologruto TA, Hannan F, Hakker I, Svoboda K, and Zhong Y. Stereotyped odor-evoked activity in the mushroom body of
Drosophila reavealed by green fluorescent protein-based $\mathrm{Ca}^{2+}$ imaging. J Neurosci 24: 6507-6514, 2004.

Wehr M and Laurent GJ. Odour encoding by temporal sequences of firing in oscillating neural assemblies. Nature 384: 162-166, 1996.

Willmore B and Tolhurst DJ. Characterizing the sparseness of neural codes. Network: Comput Neural Syst 12: 255-270, 2001.

Zars T, Fischer M, Schulz R, and Heisenberg M. Localization of a short-term memory in Drosophila. Science 488: 672-675, 2000. 